\title{
Mapping the Sea of Opportunities: Blockchain in Supply Chain and Logistics
}

\author{
Moritz Petersen, Niels Hackius, Birgit von See
}

\begin{abstract}
Driven by successful pilot projects in supply chain and logistics, Blockchain has become one of the industry's latest technology hypes. In this paper, we cut through the hype and shed light on the expectations of industry professionals towards the benefits and challenges of Blockchain. Also, we categorize current Blockchain applications that are expected to provide tangible benefits for supply chain and logistics processes. To explore such potentials, we argue that companies should gain own first-hand experiences through small-scale experiments.
\end{abstract}

\section{Introduction}

Blockchain is an emergent technology concept that enables the decentralized and immutable storage of verified transaction data. It was invented by one or more individuals going by the pseudonym of Satoshi Nakamoto [24] and has been more of an insider's tip for the longest part of its existence. After Blockchain became widely known in the financial industry around 2015, also the supply chain and logistics (SC\&L) community caught on and slowly began to realize the difference Blockchain could make for many of their processes. One major promise of Blockchain is to create transparency - every member of the network has access to the same data, providing a single point of truth [27]. Transparency throughout the entire supply chain is one of the most important and hardest to achieve improvement areas for SC\&L. It comes as no surprise that industry experts consider Blockchain to offer "enormous potential" [25] and to be able to "transform the supply chain and disrupt the way we produce, market, purchase and consume our goods" [6]. Specifically, because of the transparency promise, Blockchain might be nothing less than the "holy grail" for SC\&L [28]. Pilot projects by industry heavyweights illustrate the breadth of possibilities Blockchain offers to bring forward SC\&L processes, e.g., by eliminating extensive paperwork in shipping or making the source of foodborne outbreaks identifiable in only seconds.

However, recent studies find that logistics operators, especially small and medium-sized companies, still have little knowledge about Blockchain [17] or appear torn regarding barriers and benefits [12]. Also, since Blockchain is a foundational technology that is adopted gradually instead of sudden, it might take years until it profoundly changes the SC\&L landscape [16]. Despite the rising attention Blockchain as a new and exciting technology incurs at the moment, there is a striking gap between the huge expectations on shortterm disruptive change and ready-to-use solutions on the market. It appears fair to say that there is probably too much hype around Blockchain in SC\&L [2]. However, the hype and the exaggerated expectations should not distract from the potential Blockchain holds for the long-run. Since research on Blockchain applications is still in its infancy [33, 34], the goal of this manuscript is to cut through the hype and to shed light on the state of play of Blockchain applications in SC\&L. Specifically, we provide insights into the research questions (1) "What are the expectations towards Blockchain among SC\&L industry professionals?" and (2) "What are the hot spots of current Blockchain applications in SC\&L?". The remainder of the paper is structured as follows: First, we give an overview of the SC\&L discipline and the flows to be managed. Then, we introduce the findings of an online survey on the prospects and challenges of Blockchain in SC\&L and also highlight differences between groups of participants. Next, we categorize current Blockchain applications in SC\&L and discuss the hot spots. We conclude the paper with some limitations, further research opportunities, and an outlook. 


\section{How SC\&L Benefits From Digital Transformation}

Logistics is often solely associated with operational activities such as transportation, handling, and warehousing. However, this rather narrow and traditional perspective has been revised several times [20]. Following a more cross-functional perspective, logistics aims at ensuring the seven rights of delivery: the right product, quantity, condition, place, time, customer, and cost [30]. To achieve those targets, the operational logistics tasks are supplemented by tactical planning and controlling activities [3]. Supply Chain Management (SCM) even expands this definition of logistics and also covers the network of relationships between the entities of a supply chain [4]. Essentially, managing supply chains means managing flows between the source of the raw materials all the way to the end customer [19]. Figure 1 shows a schematic illustration of a typical supply chain. The downstream material flow through different modes of transport is accompanied by an upstream financial flow as well as a bidirectional information flow.

In today's globalized world, a product has gone through many hands and travelled a long way before it finally reaches the customer. The material flow begins with the production of raw materials and its supply to manufacturing companies. After being produced and delivered, the product is sold through retail companies. The flow ends (at least for the time being) with the delivery of the product to the customer [19]. All stakeholders within a supply chain, including logistics service providers, are traditionally focused on efficiency optimization since logistics generally is considered to be a cost center rather than a value driver [4]. The material flow is accompanied by a financial flow. In practice, material and financial flow are far from being in sync. While end consumers normally pay for the product the moment they get it, upstream supply chain partners can experience a latency of multiple months. Despite such problems, the financial flow in supply chains is a research topic lacking empirical foundation [10].

The information flow is a core resource in SCM because it offers further opportunities for supply chain optimiziation [4]. On the one hand, information flowing downstream to the next supply chain stage, e.g. about the status of a shipment, helps to better plan and syncronize upcoming activities. On the other hand, upstream information, e.g. about planned order volumes, will help to better fulfil the customers' needs. In short, up-to-date information is needed to enable transparency throughout the supply chain. This need has been articulated ever since [9], however, it is still not solved [17]. To illustrate the lack of a tightly integrated information flow with an example: When shipping a container of perishable goods from East Asia to Euro- pe, nearly 30 different organizations respectively people are involved. They exchange information on about 200 separate occasions - often through printed paperwork. It is no surprise that the cost of the paperwork processing can easily surpass 15 to $20 \%$ of the shipment costs for the ocean voyage [11].

The digital transformation offers plenty of opportunities to optimize SC\&L. This applies not only to the application of new hardware such as autonomous vehicles, but above all to the optimization of the information flow. Based on a comparative survey, digital disruption in SC\&L is expected to be high [8], however, until now the implementation of digital technologies for the benefit of SC\&L can be classified as low [7]. Central technological concepts such as platforms, data analytics, or cloud computing are not widespread at all [17]. This seems to be symptomatic since in general innovativeness in logistics is lacking behind compared to other industries like pharmaceuticals or telecommunication [32].

SC\&L experts expect further digitalization to bear cost reductions as well as increases in revenue [17]. On the one hand, cost reductions might result from digital workflows with cross-company standardized ITinterfaces. On the other hand, for example, being able to provide real-time information will increase customer satisfaction - an often neglected facet of digitalization benefits in SC\&L [14]. There are several different technologies around that might potentially play an important role in shaping future digital supply chains. One technology that promises significant advancements for information sharing in SC\&L is Blockchain.

\section{Exploring Expectations Towards Blockchain in SC\&L}

We designed and executed a web-based survey to explore how experts from the SC\&L industry evaluate Blockchain [12]. This section introduces the study's setup and the most important findings.

\subsection{Setup and Data Collection}

The survey was implemented using Typeform. We inquired the participants' stance towards Blockchain, likely adoption barriers, and the expected effect on established processes in SC\&L. We also inquired the level of general knowledge of SC\&L and Blockchain as well as job and company details.

We collected the data between April and June, 2017. Participants were mainly recruited through SC\&Lspecific social media (e.g., relevant interest groups on LinkedIn, Xing, and Meetup). The BVL (Bundesvereinigung Logistik e.V.), an international network for SC\&L experts, shared our call for participants through 

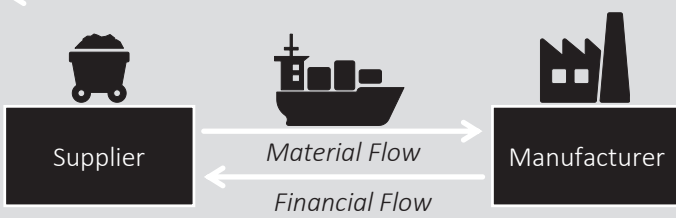

Information Flow
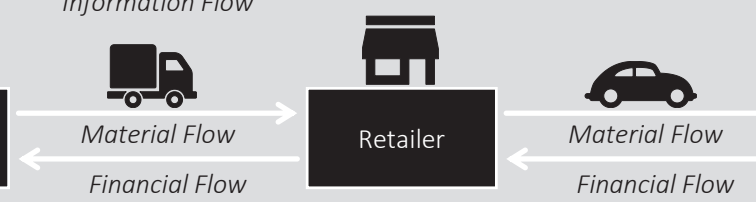

Figure 1: Schematic Illustration of Material, Information and Cash Flow within a Supply Chain
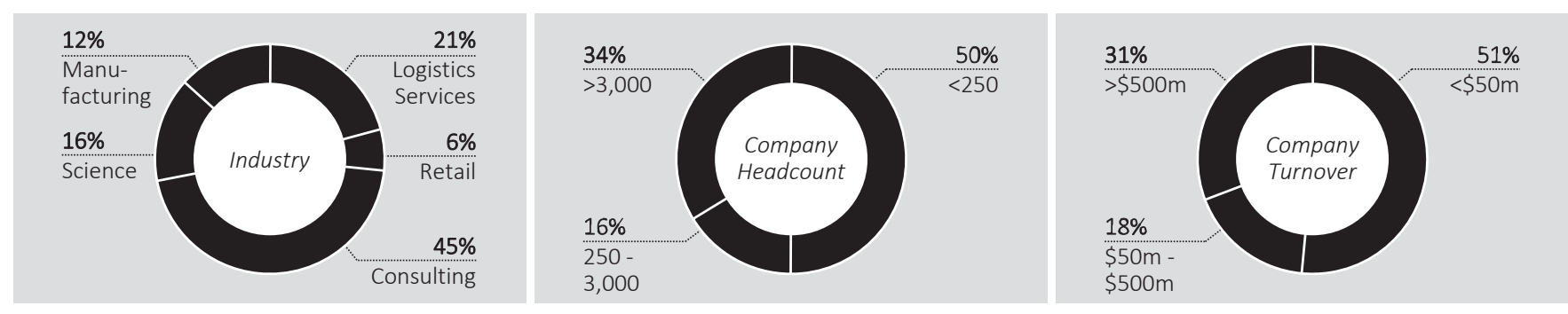

Figure 2: Overview of Participants and their Companies

their social media channels. We incentivized participation through small donations. After we concluded data collection, we donated 100 Euro to two charities on behalf of our participants.

For anonymous internet surveys, thoroughly examining the data is advised to identify careless responses that might skew the findings [23]. We excluded three out of 155 initially collected datasets due to clear answer patterns or answers that made no sense. Then, we prepared the data for statistical analyses following the guidelines of Hair et al. [13]. We used IBM SPSS Statistics 22 for the analyses.

\subsection{Findings and Discussion}

We present parts of the study's findings [12] in the following, mainly through providing the mean values of the participants' evaluations. We present more detailed findings and explore the differences between groups of participants if our analyses proved them to be statistically significant at the 5\%-level.

Figure 2 shows an overview of our 152 participants: The clear majority works in SC\&L-related consulting, followed by logistics services and sciences. All other participants work in SC\&L departments in retail or manufacturing companies. Most participants work for small and medium-sized companies with a headcount of under 250 people and an annual turnover of less than USD $50 \mathrm{~m}$. This distribution is mainly caused by a high number of participants from small consulting companies. If considered on their own, around $60 \%$ of the participants from the SC\&L companies work for large companies with over 3.000 employees and more than USD 500m turnover. In terms of their country, more than half of our participants come from Ger- many, followed by the US, Switzerland, and France. We then asked the participants about their company's stance towards Blockchain. Figure 3 summarizes the findings. $43 \%$ declare they do not look into Blockchain just yet or observe the development from a distance. $37 \%$ of the participants investigate applications, and $20 \%$ have implemented first Blockchain solutions. Again, this distribution is largely caused by consulting companies as almost three-quarters of them either investigate or implement Blockchain solutions. Looking only at the SC\&L companies, around $65 \%$ declare to be - if at all - watching from a distance. Only two of them already experiment with Blockchain technology - one start-up and one logistics services behemoth.

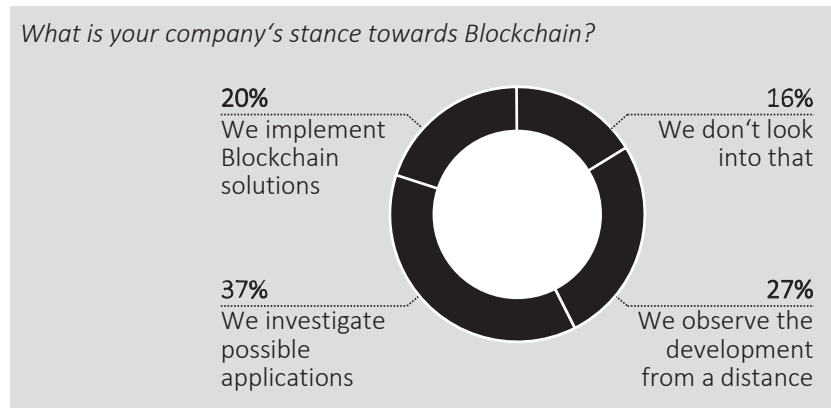

Figure 3: Company's Stance towards Blockchain

We also asked the participants about likely barriers to Blockchain adoption in the SC\&L industry. Figure 4 summarizes the findings. For $56 \%$ of the participants, regulatory uncertainty might be a barrier. Around half of the participants also refer to the fact that different parties must join forces, a lack of technological maturity, and a lack of acceptance in the industry as major 
barriers. Data security concerns (41\%), unclear benefits $(40 \%)$, and too much dependence on Blockchain operators $(28 \%)$ are also named to be likely showstoppers. Finally, we inquired the participants' overall eva-

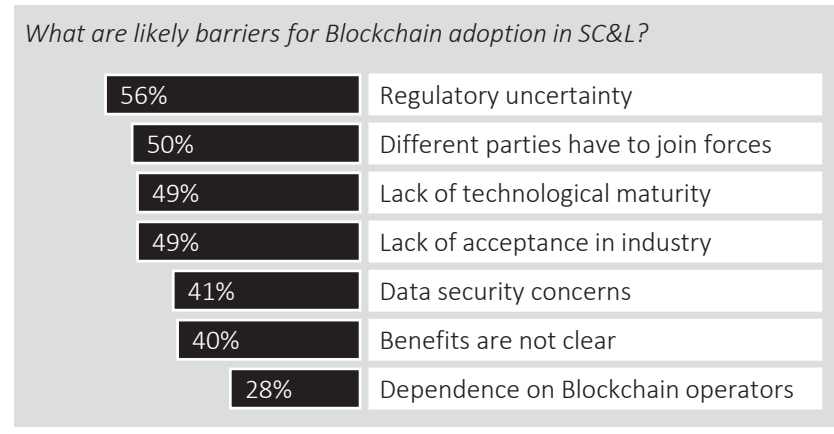

Figure 4: Barriers to Blockchain Adoption

luation of Blockchain's effect on established processes and business models in SC\&L. The findings are shown in Figure 5. With an average evaluation of 7.08, the participants believe Blockchain would have a strong effect on the industry, even though the transformation might not be as radical as the current hype leads to believe. The size of the standard deviation (1.88) proves there are skeptics and enthusiasts in the sample. However, they cannot be assigned to a specific group, like consultants or early adopters of Blockchain.

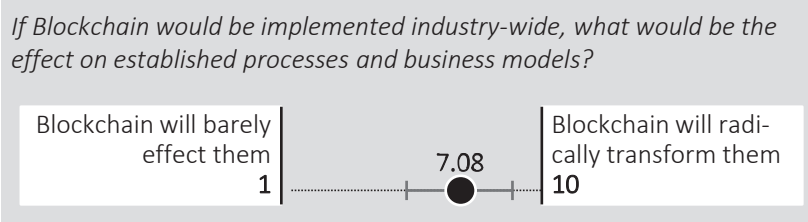

Figure 5: Blockchain's Effect on Established Processes

Further, the findings also provide insights into the perspectives of different participant groups. Taking the hierarchical level of the participants into account, our data suggests middle managers are much less enthusiastic about Blockchain than c-level executives or operational employees. They see fewer benefits and expect more showstoppers. For example, $60 \%$ of the middle managers raise concerns about data security, while only $28 \%$ of c-level executives share this reservation. An explanation for this lack of enthusiasm might lie in the better overview middle managers have of their processes and their responsibility for implementing new IT solutions (at least from the business perspective). A second differentiator is the level of Blockchain experiences. Our data suggests the more experienced participants are (e.g., exploring applications instead of just observing the development in the industry), the more positive they evaluate Blockchain. Also, their perspective on possible barriers shifts: While only one-fourth of the little-experienced participants expect the need to collaborate with different partners to be a showstopper, around $60 \%$ of participants having implementation experiences indicate a high level of collaboration and commitment is a barrier. Finally, our data suggests significant differences between SC\&L experts (working for logistics service providers, manufacturers, and retailers), on the one hand, and consultants and scientists on the other. These differences are particularly striking in terms of adoption barriers. SC\&L experts seem to have difficulties getting a clear idea of the benefits and applications, while consultants and scientists worry about the technological maturity of Blockchain.

Despite realizing the impact Blockchain might have on their industry, companies seem hesitant to dedicate resources to look into possible Blockchain applications. Our findings underline the importance of clearly outlining promising application fields. If people from a rather conservative and efficiency-focused industry, like SC\&L, are expected to buy into new technology, the benefit must be very clear. Just because something is new doesn't mean everyone gets too excited.

\section{Mapping Blockchain Applications in SC\&L}

To shed light on the second research question pertaining to current applications of Blockchain for the benefit of SC\&L processes, we conducted a thorough review of relevant practitioner journals, newspapers, and social media. We introduce our findings in the following sections.

\subsection{Overview of Applications}

We identified 49 different applications of Blockchain technology in the SC\&L context publically known until the end of September 2017. In general, the applications range from mere announcements of future collaborations to Blockchain services already available on the market. We collected sufficient information and classified each case according to criteria like time of the announcement, type of project, type of companies involved, and several content-related criteria. Figure 6 illustrates the growth of known applications over time. To our knowledge, the first true SC\&L application was announced in April 2015: The startup Everledger uses Blockchain to combat fraud in diamond supply chains. Since January 2016, the number of startups in this field constantly increased. During the summer of 2016, the first corporate projects were made public - often a collaboration with a consultancy company or a technology provider like IBM. The number of these projects constantly grew, especially during the second and third 
quarter of 2017. A less common form is joint efforts by startups and corporations: In these projects, the corporations often provide the testbed for solutions developed by the startups.

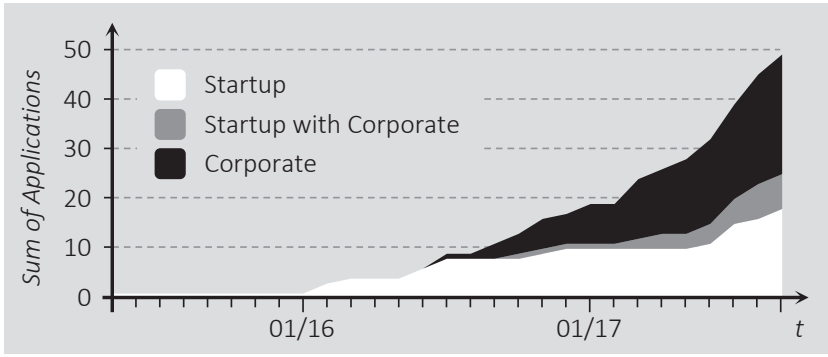

Figure 6: Growth of Blockchain Applications in SC\&L over Time

To explore existing hot spots, we matched the applications with the corresponding flows of SC\&L: material flow, information flow, and financial flow. Figure 7 provides an overview. Not surprisingly, almost all applications aim at improving the information flow. Three major clusters emerge. The first cluster is "Product Tracking". Such applications are located at the intersection of information flow and material flow as they concentrate on providing status information about shipments or other logistics objects. The second cluster "Product Tracing" mostly pertains to the information flow as these applications focus on tracing back the origin of a product. The third cluster "Supply Chain Finance" is located at the intersection of all three flows. Such applications deal with automated payment processing depending on the status of a shipment, e.g., through smart contracts. In the following, we will further introduce the clusters and exemplary applications.

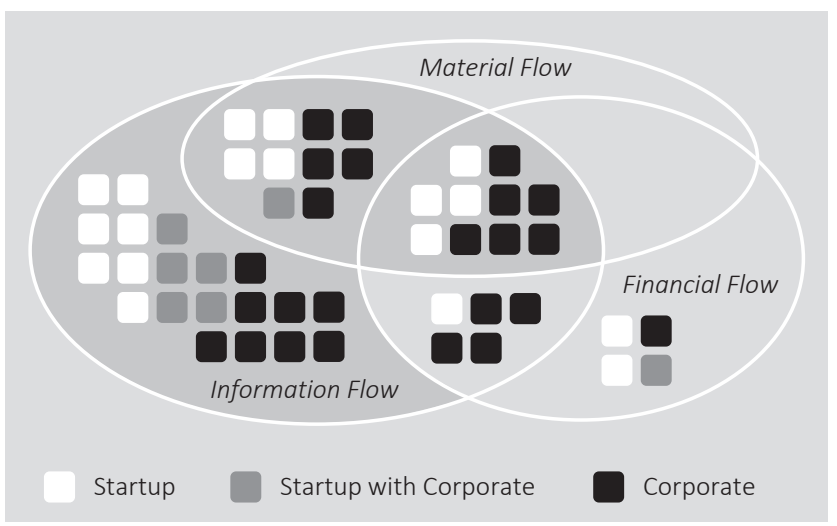

Figure 7: Matching Applications with SC\&L Flows

\subsection{Application Cluster "Product Tracking}

In SC\&L, the tracking of cargo or loading tackle is necessary to enable the supply chain partners to anti- cipate necessary changes. Deviations that may require such changes can be caused by missing or incorrect documents, but also by scheduling changes, handling errors, or newly available sensor data. The decentralized nature of Blockchain allows to easily share data across company borders and, moreover, to trust these records to a certain extent. Take handling delays of sea freight containers as an example: Depending on movement timings written into the Blockchain, downstream transportation companies or production planners are able to adjust their plans. Sharing such data across the supply chain has been possible for decades - at least in theory. However, trust issues, a lack of interoperability, and many non-digitized sub processes acted as roadblocks.

Among the screened applications in the application cluster "Product Tracking", two seem especially notable. One is the troika of the chemical industry corporation BASF, the fintech startup Quantoz, and the Dutch startup Ahrma: The consortium's intention is to make the supply chain more visible by capturing and sharing sensor data in near real time. It works on a sensor-equipped pallet that writes data into a private Blockchain accessible by all partners along the way [18]. Besides the location tracking, motion and temperature sensors enable the detection of mishandling along the supply chain. The full record not only enables suitable reactions but also provides a common database - an immutable truth visible to all parties - which allows holding the respective partner responsible. Similarly, at a proof-of-concept level but still noteworthy, is a joint project by the software behemoth Microsoft and the RFID integrator Mojix. Whilst Microsoft provides the software knowledge and infrastructure, Mojix has knowledge about implementing RFID scanning technology in the textile retail sector. In the project, the companies intend to close the gap between the different databases suppliers, retailers, and carriers create. This would enable them to make their supply chain even more agile by making or updating decisions as soon as new data becomes available [5]. Moreover, the database also allows for analytics to increase efficiency. Whilst the current implementation of this project is private, a long-term goal is to create end-to-end provenance checks for retail customers by putting parts of the data on a public Blockchain [21].

\subsection{Application Cluster “Product Tracing}

Provenance in SC\&L is important, not only to endconsumers but regardless of the position in the supply chain, to prove the authenticity of a shipment or the origin of a product. The second cluster contains applications that aim to address this problem. The decentralization and public access models of Blockchain enable an immutable record keeping down to the product level. Blockchain applications in this space make 
use of this by tracing back along the information flow to certain verified data points. Tracing a pharmaceutical product back to its original manufacturer to assess its genuineness can be crucial in developing countries where a pharmacist might not be able to trust the wholesaler. Also, it might be required to find out at which points the product has been handled. In a food supply chain, it can become necessary to inform upstream supply chain participants about contaminated food. Today, the traceability "from farm to fork" is still a more theoretical idea and is limited by existing technologies [1].

With regards to the application cluster "Product Tracing", three applications seem especially notable. BHP Billiton, one of the world's largest mining companies, has created an application to trace the provenance of geological samples all over the world. Such samples are extremely valuable, especially because it is so costly to obtain them. Until recently, the samples were traced through spreadsheets and e-mails. Now, the original sampling location and further handling data is immutably recorded on the public Ethereum Blockchain [29]. Metadata added later is linked to this record using a decentralized storage system (IPFS) that also allows to attribute the changes to a certain owner. In case of a lost sample, it can now always be traced back to the last owner. Furthermore, the data can be accessed through dedicated smartphone applications by all parties involved.

Two other cases both involve the food industry: In a pilot project, the consulting company Ernst \& Young and the Italian agriculture startup Ez lab announced the "wine Blockchain" in April 2017. Built on the public Ethereum Blockchain, it is possible to trace back through each step of the supply chain from the endconsumer to the production site. The intention is to safe-guard against counterfeit wine, but also to handle recalls and customer requests with a common identifier. The second case is a trial by an Australia-based partnership of the $\mathrm{CBH}$ group, a grain exporter, and the startup AgriDigital. CBH hopes to gain a competitive advantage in Asia by providing a proof that their exported oat grains originally is from Australia [35].

\subsection{Application Cluster "Supply Chain Finance}

The third cluster "Supply Chain Finance" contains Blockchain applications that approach financial issues from different angles. Despite its undisputed importance, Supply Chain Finance is still an emerging field of research. It mostly covers the optimization of working capital (accounts payable, receivable, and inventories) among the members of a supply chain, including financial institutions [10]. Since Blockchain was invented through establishing the cryptocurrency Bitcoin, its underlying principles are a natural fit for Supply Chain Finance. In short, it is expected that Blockchain will enable faster payments, will lower the transaction costs (especially in multi-currency supply chains), and mitigate the risk of fraud [15].

One application is an initiative set up by IBM, the trading house Trafigura, and the investment bank Natixis. It focuses on digitizing crude oil transactions in the US through the Blockchain [26]. In short, it provides the buyer and the seller of crude oil along with their respective banks with the same, verified and tamperproof data about a transaction. The consortium hopes to improve transparency, security, and efficiency of all aspects of crude oil transactions. Another application was announced by Foxconn, one of the largest electronics manufacturers and important supplier of Apple. Together with partners, they launched a Blockchainpowered platform that provides small manufacturers upstream the supply chain with working capital [22]. Such companies need working capital to bridge the gap between paying their workers and settling of invoices. Sometimes, these gaps can be big enough to cause sufficient financial trouble for small companies to close operations. For big corporations downstream the supply chain (such as Foxconn) it is essentially a risk management measure to ensure their multiple levels of suppliers is financially sound and has access to working capital in the case of a disruption (like a fire in a factory or the loss of an important customer). A similar application for the pharmaceutical supply chain was announced by IBM and the Chinese company Hejia [31]. Today, small retailers are paid 60 to 90 days after the medicine has been delivered to a hospital. Through putting such transactions on the Blockchain, the consortium hopes to reduce this gap and, thus, the need for working capital. Essentially, they try to further integrate the material, information, and financial flows of the supply chain.

\subsection{Discussion}

The three clusters introduced above give an overview of current hot spots of Blockchain applications in SC\&L. Yet, they are just a rough categorization of the diverse efforts taking place at the moment. Often, the announcements available contain only sparse details and only give an overview of the project. We can see, however, that the applications aim at augmenting and automating the material and financial flow. Increases in efficiency and optimization of business processes are not a side benefit, but make this change possible, because typically upgrading the IT infrastructure alone is not an option.

As the application landscape permanently evolves, new clusters or sub-clusters might soon emerge. One subcluster of Supply Chain Finance would probably focus on the initial coin offerings popping up in the last months. In the area of tracking, it is likely that more applications emerge that can automate the steps to 
be taken in case of a deviation. Whilst it seems obvious that tracing could largely merge with trackingtype applications, we still expect specialized use-cases for certification tracking, e.g. for organic or fair trade food labels. Overall, the further adoption of the Internet of Things, especially the widespread use of cheap sensors, will be a major driver for Blockchain applications in SC\&L. In terms of IT in SC\&L, this will imply a whole world of new interfaces as well as data handling to be taken care of.

\section{Limitations and Future Research}

Our studies hold some limitations that have to be observed for interpreting their findings. First, the sample of the online survey is not representative of the underlying population of SC\&L companies. It is likely that our survey mostly attracted professionals that already have a basic knowledge about Blockchain. Second, our research on applications does only cover cases where suitable information was publically available. From many discussions within the industry, we know that a number of SC\&L companies are working on Blockchain solutions without sharing this information with the public. Also, we only included cases that were announced until September 30th, 2017. Since Blockchain is a hot and evolving topic at the moment, the number of applications is likely to grow, and also the hot spots might shift. Thus, future research should take this into account and examine which applications progressed from a mere announcement to a solution available on the market. It further appears valuable to more closely examine the applications from a technological point of view and, among other issues, explore which Blockchain implementations are used, who runs the nodes, and which consensus mechanisms are used.

\section{Conclusions}

This paper took a closer look at Blockchain applications in SC\&L. Doing so, we intended to cut through the current hype and shed light on the state of play. First, we presented findings from an online survey regarding the expectations towards Blockchain among SC\&L industry professionals. Taken together, our respondents realize the impact Blockchain might have on the industry. However, especially the rather conservative logistics companies seem hesitant to dedicate resources to look into possible Blockchain applications. Second, we investigated the hot spots of current Blockchain applications in SC\&L. We found 49 relevant cases and identified three major clusters: Tracking of products during their journey through the supply chain, tracing back the product origin, and supply chain finance. However, also the applications outside of these main clusters exemplify the size of Blockchain's sea of opportunities for SC\&L. Our findings show small-scale experiments with Blockchain applications are vital to understand the barriers and benefits of Blockchain and, thus, to be able to navigate the sea of opportunities. Even though the change comes slower than many expected still a couple of months ago, SC\&L companies should engage in experiments to find out if and how Blockchain could be of use for their own business. Our research might provide a starting point to explore the opportunities Blockchain offers for SC\&L.

\section{Literature}

[1] Myo Min Aung and Yoon Seok Chang. Traceability in a food supply chain: Safety and quality perspectives. Food Control, 39(1):172-184, 2014.

[2] Steve Banker. Blockchain in The Supply Chain: Too Much Hype. Forbes, September 1st, 2017.

[3] Donald Bowersox, David Closs, M. Cooper, and M. Bixby. Supply Chain Logistics Management. McGraw-Hill/Irwin, Boston, MA, 3rd edition, 2010.

[4] Martin Christopher. Logistics and Supply Chain Management. Financial Times Prentice Hall, Harlow, 4th edition, 2011.

[5] Michael del Castillo. Microsoft Unveils Project Manifest, A Plan For Blockchain Product Tracking. CoinDesk, January, 2017.

[6] Ben Dickson. Blockchain Has the Potential to Revolutionize the Supply Chain. Techcrunch, November 24th, 2016.

[7] Mathieu Dougados and Boris Felgendreher. The Current and Future State of Digital Supply Chain Transformation. New York, 2016.

[8] Frank Farrall, Claire Harding, Robert Hillard, John O'Mahony, Ric Simes, and Damien Tampling. Digital disruption - Short fuse, big bang? Deloitte Touche Tohmatsu, 2012.

[9] Stanley E. Fawcett and Gregory M. Magnan. The Rhetoric and Reality of Supply Chain Integration. International Journal of Physical Distribution \& Logistics Management, 32(5):339-361, 2002.

[10] Luca Mattia Gelsomino, Riccardo Mangiaracina, Alessandro Perego, and Angela Tumino. Supply Chain Finance: A Literature Review. International Journal of Physical Distribution \& Logistics Management, 46(4):348-366, 2016.

[11] Tom Groenfeldt. IBM And Maersk Apply Blockchain To Container Shipping. Forbes, March 5th, 2017.

[12] Niels Hackius and Moritz Petersen. Blockchain in Logistics and SCM: Trick or Treat? In Proceedings of Hamburg International Conference of Logistics, 2017.

[13] Joseph F. Hair, William C. Black, Barry J. Babin, and Rolph E. Anderson. Multivariate Data Analysis. Prentice Hall, Upper Saddle River, 2009.

[14] Kai Hoberg and Knut Alicke. How SC4.0 will Enhance the Customer Experience. Supply Chain Management Review, (September/October):28-37, 2016.

[15] Erik Hofmann, Urs Magnus Strewe, and Nicola Bosia. Supply Chain Finance and Blockchain Technology: The Case of Reverse Securitisation. Springer, Cham, 2017.

[16] Marco Iansiti and Karim R. Lakhani. The Truth About Blockchain. Harvard Business Review, 95(1):118-127, 2017.

[17] Wolfgang Kersten, Mischa Seiter, Birgit von See, Niels Hackius, and Timo Maurer. Trends and Strategies in Logistics and Supply Chain Management - Digital Transformation Opportunities. DVV Media Group, Hamburg, 2017. 
[18] Susan Lacefield. Pilot project connects blockchain and ßmartpallets. CSCMP's Supply Chain Quarterly, September, 2017.

[19] Douglas M Lambert. Supply Chain Management: Processes, Partnerships, Performance. Supply Chain Management Institute, Sarasota, 3rd edition, 2008.

[20] Paul D Larson, Richard F Poist, and Árni Halldórsson. Perspectives on Logictics vs. SCM: A Survey of SCM Professionals. Journal of Business Logistics, 28(1):1-24, 2007.

[21] Jim Manning. Microsoft's Project Manifest: BlockchainBased Product Tracking. ETHNews, January, 2017.

[22] Jennifer McKevitt. Foxconn turns to blockchain to help suppliers avoid financial disruption. SupplyChainDIVE, March 14th, 2017.

[23] Adam W. Meade and S. Bartholomew Craig. Identifying Careless Responses in Survey Data. Psychological Methods, 17(3):437-455, 2012.

[24] Satoshi Nakamoto. Bitcoin: A Peer-to-Peer Electronic Cash System. 2008.

[25] Kevin O'Marah. Blockchain For Supply Chain: Enormous Potential Down The Road. Forbes, March 9th, 2017.

[26] Luke Parker. Hyperledger used to track US oil on a blockchain. Brave New Coin, March 30th, 2017.

[27] Marc Pilkington. Blockchain Technology: Principles and Applications. In F. Xavier Olleros and Majlinda Zhegu, editors, Research Handbook on Digital Transformations, pages 1-39. Edward Elgar Publishing, 2016.

[28] Nathaniel Popper and Steve Lohr. Blockchain: A Better Way to Track Pork Chops, Bonds, Bad Peanut Butter? New York Times, March 4th, 2017.

[29] Pete Rizzo. World's Largest Mining Company to Use Blockchain for Supply Chain. Coindesk, September 23rd, 2016.

[30] Roy D Shapiro and James L Heskett. Logistics Strategy: Cases and Concepts. West Pub. Co, St. Paul, MI, 1985.

[31] Aaron Stanley. IBM Ramps Up China Blockchain Work With Supply Chain Trial. Coindesk, April 11th, 2017.

[32] Stephan M. Wagner. Innovation Management in the German Transportation Industry. Journal of Business Logistics, 29(2):215-231, sep 2008.

[33] Jesse Yli-Huumo, Deokyoon Ko, Sujin Choi, Sooyong Park, and Kari Smolander. Where Is Current Research on Blockchain Technology? - A Systematic Review. PLoS ONE, 11(10):1-27, 2016.

[34] J. Leon Zhao, Shaokun Fan, and Jiaqi Yan. Overview of Business Innovations and Research Opportunities in Blockchain and Introduction to the Special Issue. Financial Innovation, 2(28):1-7, 2016.

[35] Wolfie Zhao. Australia's Biggest Grain Exporter Trials Blockchain Tracking System. Coindesk, August 3rd, 2017. 